\title{
Krige Method Application for the Coverage Analysis of a Node-B in a WCDMA Network
}

\author{
Jazmín Ponce-Rojas, Sergio Vidal-Beltrán, José Luis López-Bonilla, Montserrat Jimenez-Licea \\ Maestría en Ciencias en Ingeniería de Telecomunicaciones, Escuela Superior de Ingeniería Mecánica y Eléctrica, \\ Instituto Politécnico Nacional, México City, México \\ E-mail: ponce_jaz7@hotmail.com, sergiovidalb@mac.com \\ Received January 17, 2011; revised February 19, 2011; accepted February 21, 2011
}

\begin{abstract}
This paper shows the procedure and application of the Krige Method (or Kriging) for the analysis of the power level radiated by a Base Station (also called Node B), through a group of samples of this power level, measured at different positions and distances. These samples were obtained using an spectrum analyzer, which will allow to have georeferenced measurements, to implement the interpolation process and generate coverage maps, making possible to know the power level distribution and therefore understand the behavior and performance of the Node B.
\end{abstract}

Keywords: Interpolation, Krige, Node B, WCDMA

\section{Introduction}

At the present time WCDMA (Wideband Code Division Multiple Access) is a medium access technique implemented in many countries around the world, including Mexico. This technique allows the user to occupy the entire bandwidth in any time, because identifying information of each mobile station through codes, allowing reliable transmission within the same channel not separated in time or frequency. WCDMA provides high transfer rates, efficient support of asymmetric traffic, transmission using packet switching through the radio interface and high efficiency in spectrum use.

The Base Station (BS), also known as Node B, is part of the UMTS Terrestrial Radio Access Network (UTRAN). The Node B has as main tasks make the transmission and reception of radio signal, filtering of the signal, amplification, modulation and demodulation of the signal and be an interface to the Radio Network Controller (RNC) [1]. The Common Pilot Channel (CPICH) transmits a carrier used to estimate the channel parameters; is the physical reference for other channels. It is used to control power, consistent transmission and detection, cannel estimation, measurement of adjacent cells and obtaining of the Scrambling Code (SC) [2].

The Krige Method is an interpolation technique based in a sample regression, which are irregularly spaced, to predict unknown values from known values. It is a fam- ily of generalized algorithms for least squares that from a set of observations provides the optimal linear predictor for the variable in any position.

This method was developed by Daniel G. Krige in an attempt to predict more accurately the mineral reserves. In recent decades the Krige Method has become a fundamental tool in the field of the geostatistics because founded the basis of linear geostatistical. It is a geostatistical method for estimating points that using a variogram model to obtain data. It is based on the premise that the spatial variation continues with the same pattern.

The variogram or semivariogram is a tool that allows analyzes the spatial behavior of a variable on a defined area, resulting in the influence of data at different distances. From the data provided by the theoretical variogram the estimation is performed by the method of Krige. The semivariogram is a measure of how similar is the points in the space when they are farther apart. To develop a variogram first requires creating an experimental variogram based on the selected simple, and choose a theoretical variogram that fit the experimental, because this is not a function where we can make interpolations.

The spatial variations correlated are treated in functions as the variogram, which show the information to optimize the weights and choose radios precise data search. Known the variogram and the original observations, you can get a set of achievements to show the range of possible values. 
There are three variants of Kriging depending on the assumptions made with respect to the mean or average value of samples:

- Simple Krige

- Ordinary Krige

- Universal Krige [3].

\section{Methodology}

The interpolation method of Krige facilitates the task of analyzing the distribution of power radiated by an antenna, it is sufficient to take a series of grab samples with the proper equipment (which is capable of obtaining the geographic coordinates of the location and analysis parameters) to create an experimental variogram, and based on it make the most appropriate approach to the theoretical variogram to perform interpolation and move from discrete samples to a continuous measurement map.

The semivariogram provides information of the spatial behavior of a variable. To quantify the degree and scale of spatial variation is necessary adjusting a function that describes the behavior of the variable. The adjustment of a function allows extract a set of parameters that are used for geostatistical interpolation (Kriging) and define the extent and scale of the spacing variation. These parameters are the range $(\mathrm{A} 0)$, the nugget $(\mathrm{C} 0)$, the sill $(\mathrm{C} 0+\mathrm{C})$, and the proportion of the variance given to the space $(\mathrm{C} /$ $\mathrm{C} 0+\mathrm{C})$, often expressed as a percentage.

The range (A0) is the distance at which semivariance stops increasing. Therefore range indicates the distance from which samples are spatially independent of each other. The nugget $(\mathrm{C} 0)$ is the variance not explained by the model, and is calculated as the intercept with the Y axis. Also known as error of variance, because the variance of two points separated by zero meters should be zero. That is why this variance is usually indicating a smaller scale variability of the sampled. The maximum semivariance found between pairs of points is known as Sill. The ratio $\mathrm{C} / \mathrm{C} 0+\mathrm{C}$ gives us the grade of spatial variation, and therefore the grade of uncertainty when interpolating points in the space. A high ratio indicates a very predictable spatially variable in Figure 1 [4].

The measurement procedure was performed in the frequency band $\mathrm{V}$, which has a central value of $887.5 \mathrm{MHz}$, adequate in Mexico to make measurements of WCDMA. The measurements were made with a spectrum analyzer, BTS Master MT8222A, made by ANRITSU. El BTS Master can measure the performance of Node B by connecting directly to the Node B equipment or through the air by connecting an antenna. To measure a signal WCDMA in air, you must connect the appropriate antenna to the frequency band to be measured. As for the location infor-

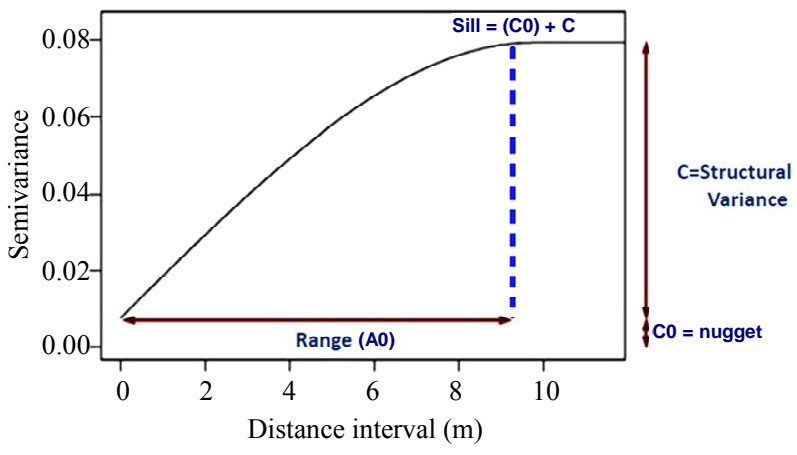

Figure 1. Variogram parameters for setting.

\begin{tabular}{|lcl}
\hline LONGITUDE & LAIITUDE & CPICH \\
-99.13472222 & 19.49777778 & -82.553 \\
-99.13472222 & 19.49777778 & -80.167 \\
-99.13472222 & 19.49777778 & -83.676 \\
-99.13472222 & 19.49805556 & -88.068 \\
-99.13472222 & 19.49833333 & -84.118 \\
-99.13472222 & 19.49833333 & -85.757 \\
-99.13472222 & 19.49833333 & -88.932 \\
-99.13472222 & 19.49861111 & -86.631 \\
-99.13472222 & 19.49861111 & -83.616 \\
-99.13472222 & 19.49888889 & -79.743 \\
-99.13472222 & 19.49916667 & -82.326 \\
-99.13472222 & 19.49944444 & -86.039 \\
-99.13472222 & 19.49972222 & -84.787 \\
-99.13472222 & 19.49972222 & -85.6 \\
-99.13472222 & 19.49972222 & -87.987 \\
-99.13472222 & 19.50027778 & -81.967 \\
-99.13472222 & 19.50027778 & -81.457 \\
-99.13472222 & 19.50083333 & -86.443 \\
-99.13472222 & 19.50083333 & -88.63 \\
-99.13472222 & 19.50111111 & -89.686 \\
-99.13472222 & 19.50111111 & -91.257 \\
-99.13472222 & 19.50166667 & -89.88 \\
-99.13472222 & 19.50166667 & -93.867 \\
-99.13472222 & 19.50166667 & -91.075 \\
-99.13472222 & 19.50166667 & -88.946 \\
-99.13472222 & 19.50194444 & -92.528 \\
-99.13444444 & 19.49583333 & -59.089 \\
-99.13444444 & 19.49583333 & -64.401 \\
-99.13444444 & 19.49583333 & -61.553 \\
-99.13444444 & 19.49583333 & -61.553 \\
-99.13444444 & 19.49583333 & -59.87
\end{tabular}

Figure 2. Data file format.

mation of each measurement requires a GPS antenna (Global Position System) [5].

The power level of the CPICH is the parameter of interest because it allows channel estimation. Therefore, generates a text file as shown in Figure 2, to start the interpolation process.

Later, entering data into the software "EasyKrig" through a text file as shown in Figure 1, data are plotted so that the geographical coordinates defined the axes, and the power level determines the color which represents the sample as shown in Figure 3. In the next step, the experimental variogram is constructed depending on the variation of the mathematical expectation of the values obtained, to determine the influence of data at different distances, as shown in Figure 4.

There are different space-temporal statistical models to obtain the experimental variogram, as: the exponential, Gaussian, Bessel, linear, to name a few. The most appropriate model for the results of the analysis of performance of a Node B is the empirical model "exponential Bessel" since the Bessel function of exponential cor- 
relation is one of the most flexible for general use. The experimental variogram is shown in Figure $\mathbf{5}$ as a red line.

Based on the minimum sum of squares, or if you have prior information of the variable behavior, can be done manually adjusting the semivariogram models. In this way you can set the nugget, the sill or the range, depending on the type information that has the variable, also adjust the parameters, which have no information.
To minimize the variance of the mean square error of estimate is necessary to have a function that describes the behavior of the phenomenon discussed, as an interpolation between the points of the experimental variogram does not guarantee the existence and uniqueness of the solution of the Kriging system, that is why, based on the experimental variogram, we construct a theoretical variogram that resembles the actual behavior of the variable through the least squares approximation.

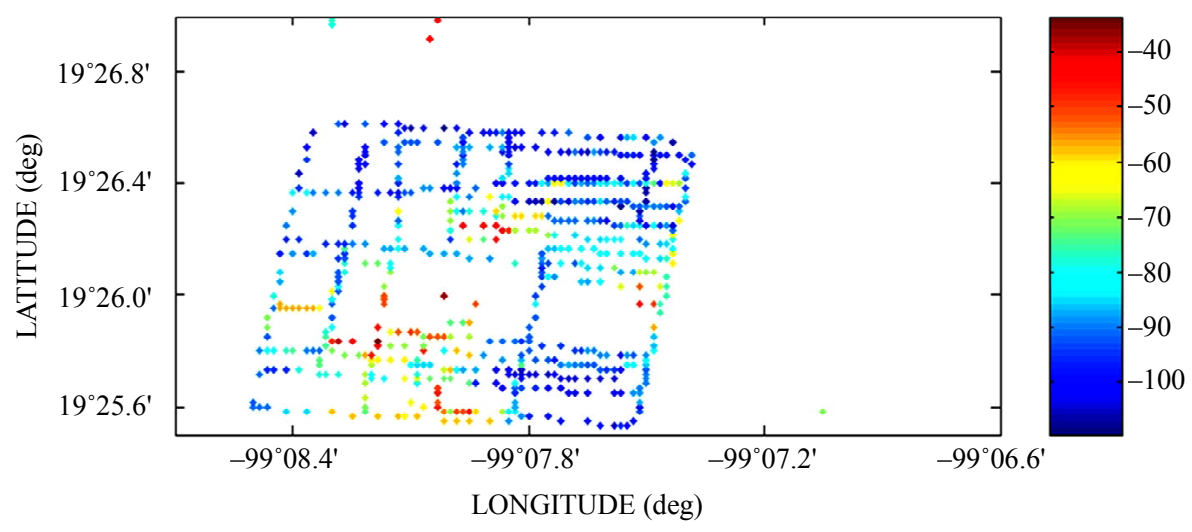

Figure 3. Location of point measurements.

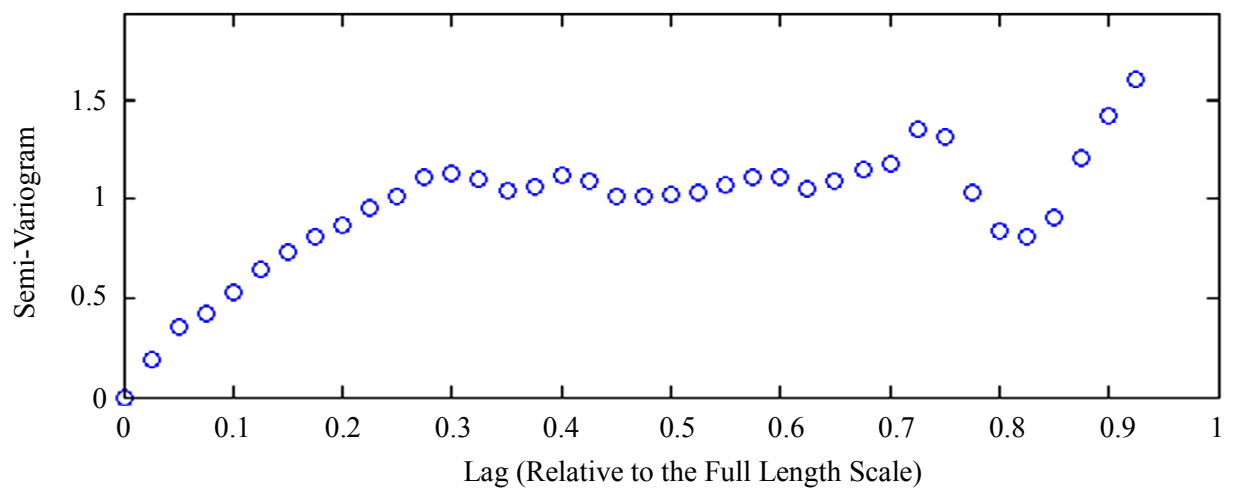

Figure 4. Experimental variogram.

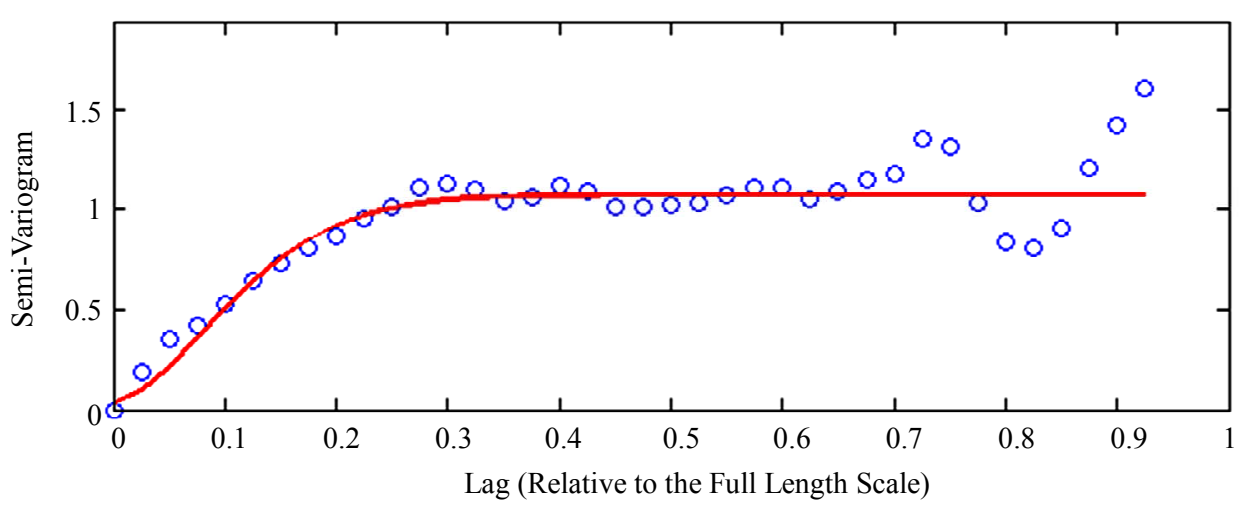

Figure 5. Theoretical variogram from LSQ. 
The Figure 6 is obtained using Ordinary Krige, because the mean value is not known, and we know that the value is not constant throughout the study area, but locally can be considered constant; because measurements are made at a distance will be very similar to those made in the vicinity of that point.

Finally, it makes the validation step shown in Figure 7, to be sure that the mean square error is within the acceptance region to eliminate any doubt with respect to reliability, efficiency, consistency, sufficiency and unbiasedness of the power level estimator for proper analysis of the performance of a Node B.

\section{Results}

As a statistical method each map generation to will be as different as theoretical variograms can be constructed. Obviously, this will depend on the behavior of data. When making a large number of samples concentrated in a given space, as is shown in Figure 8, has a behavior which is very close to the experimental way, as is shown in Figure 9.

Consequently, the approximation error Q will be very small and is within the expected range in the Gaussian bell, as shown in Figure 7.

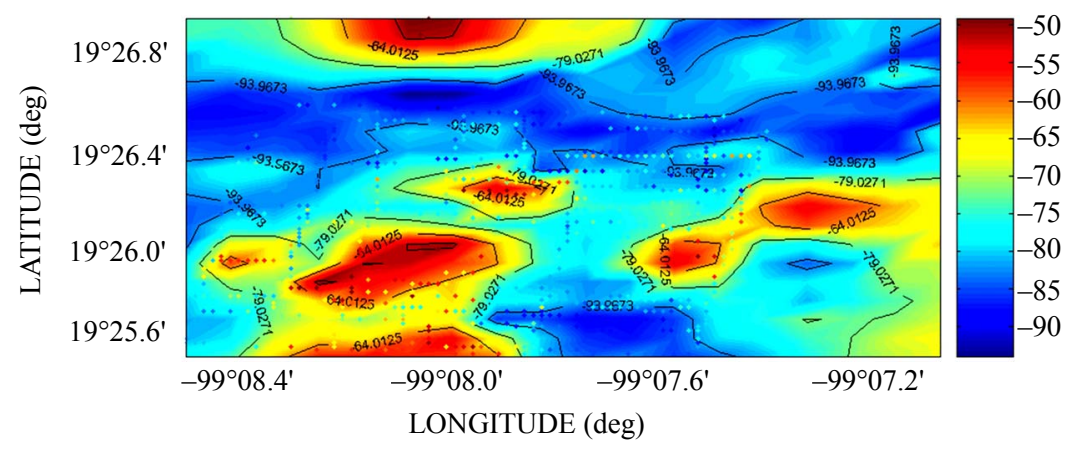

Figure 6. Coverage map.

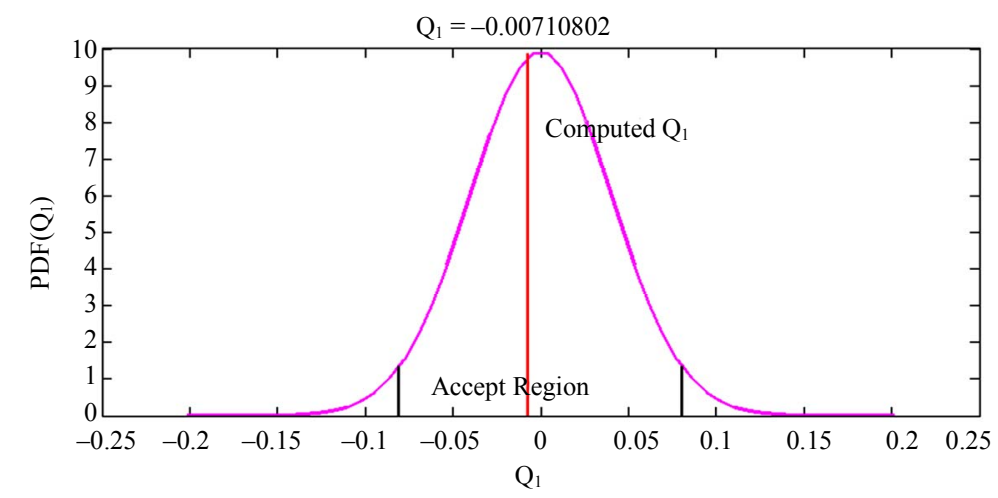

Figure 7. Validation of the interpolation process.

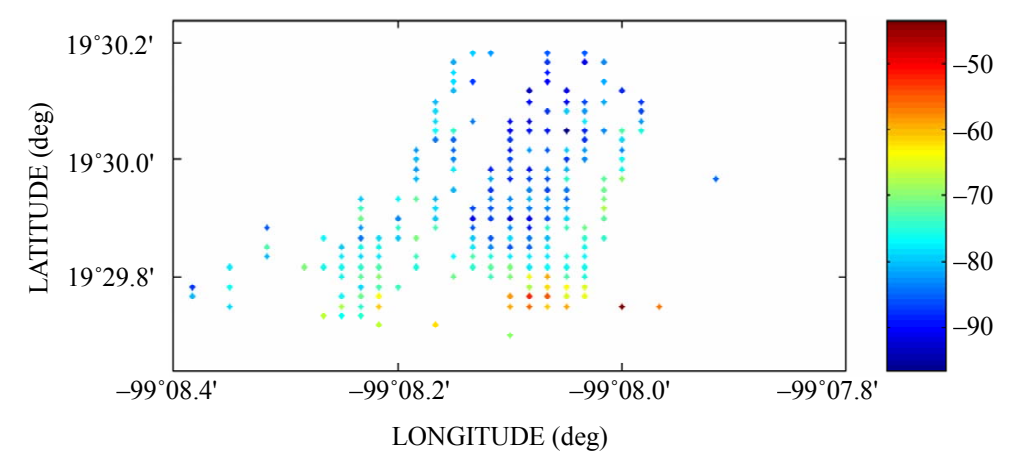

Figure 8. Location of 592 samples. 
When preset conditions are met, coverage map is properly constructed (Figure 6), and therefore is very clear assessment of the location of the Base Station, the obstacles to the propagation, the attenuation of the signal as a function of distance, the area in which the received signal is reasonably good.

Then there were three cases showing variations in the results.

In the first case were obtained 592 point samples, with a minimum value of power level of $-95.9 \mathrm{dBm}$ and maximum power measurement of $-43.8 \mathrm{dBm}$. The distribution of these measurements is shown in Figure 8. The greatest concentration is in the center of the study area, therefore be as nearby the power values measured do not vary drastically.

Although the range between the highest and the lowest power levels very ample, the large number of samples allows have a semivariogram with a fairly predictable behavior, and therefore adjust the theoretical semivariogram, as shown in Figure 9.

The efficiency of the estimate is checked through the validation process, getting the error of approximation $Q$ is within the acceptance region as shown in Figure 10.
Having a large number of samples is achieved that the area where the coverage map applies is very similar to the total test area as is shown in Figure 11.

If the samples are few, but are concentrated in a specific area of the measuring area, is possible to obtain reliable results, but only within that space, as shown in Figure 15, coordinates have smaller increments, because the data provided are sufficient to know only the power level in the vicinity of the points. Locate the Base Station is not an easy task as it is not the interpretation of the maps. This case is illustrated in Figures 12 to $\mathbf{1 5}$.

For this case were obtained 120 samples, with maximum power level of $-69.5 \mathrm{dBm}$ and a minimum of $-97.9 \mathrm{dBm}$. The distribution of samples is more irregular as shown in Figure 12.

Due of the greater distance between samples the similarity between them is less and therefore adjustment of the experimental semivariogram is more difficult as shown in Figure 13.

The approximation error is greater than in the previous case but is in the region of acceptance as shown in Figure 14.

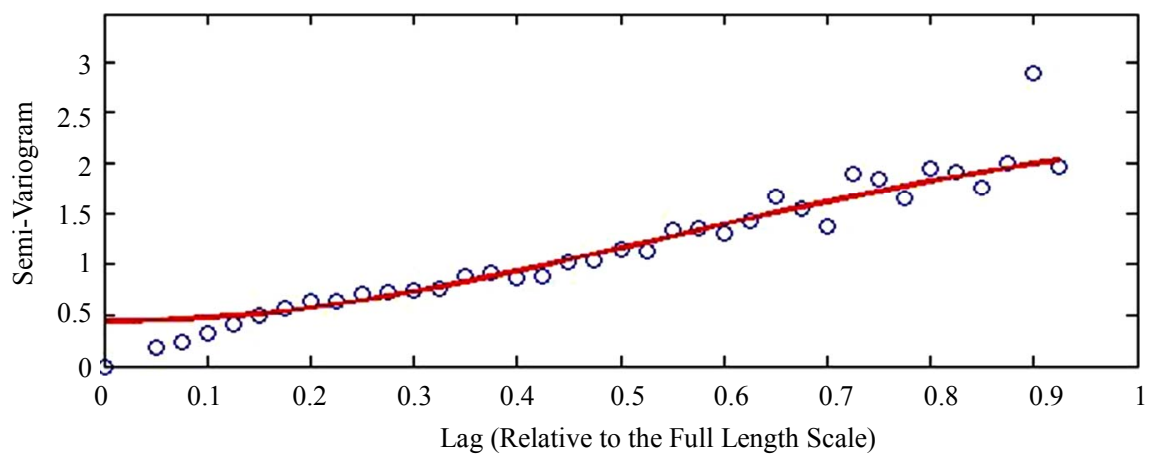

Figure 9. Setting theoretical semivariogram to experimental for 592 samples.

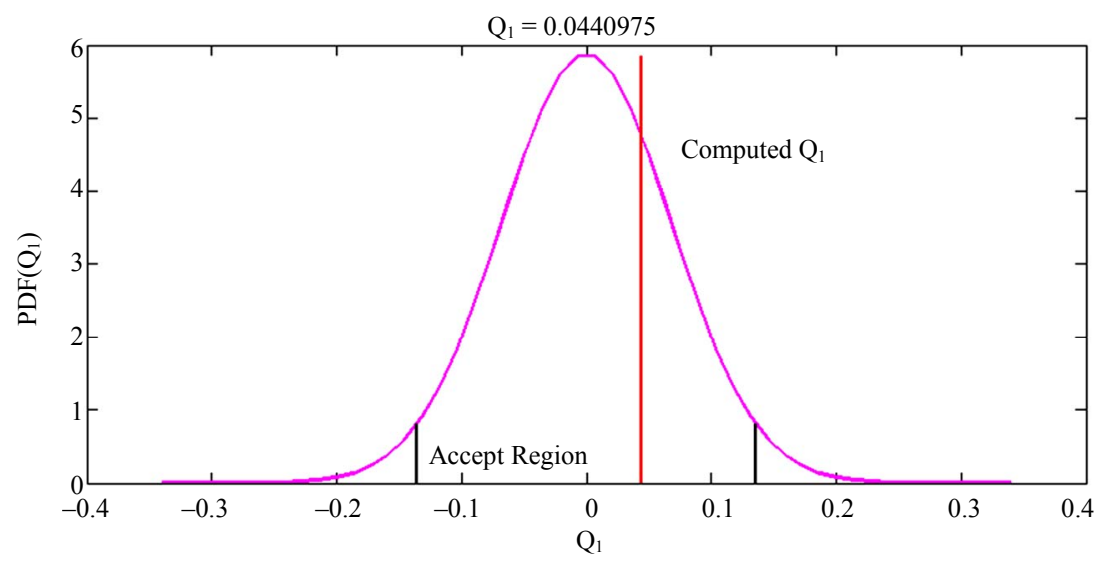

Figure 10. Validation procedures for 592 samples. 


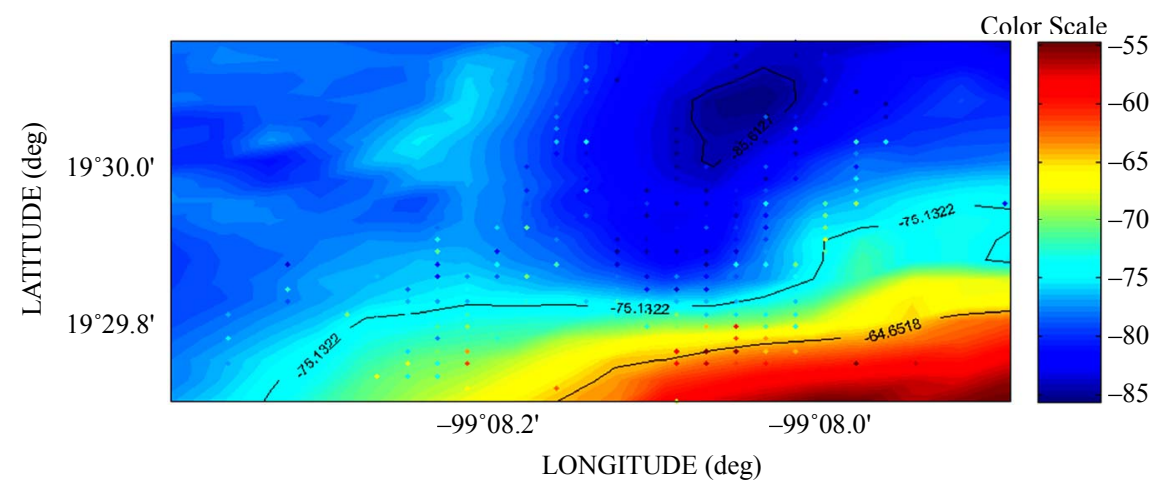

Figure 11. Coverage map for 592 samples.

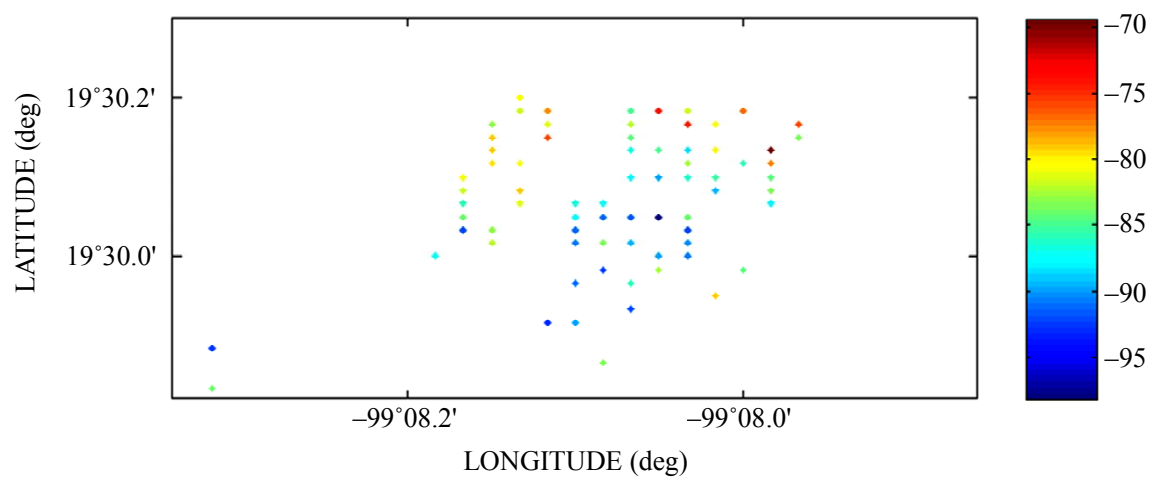

Figure 12. Location of 120 measurements.

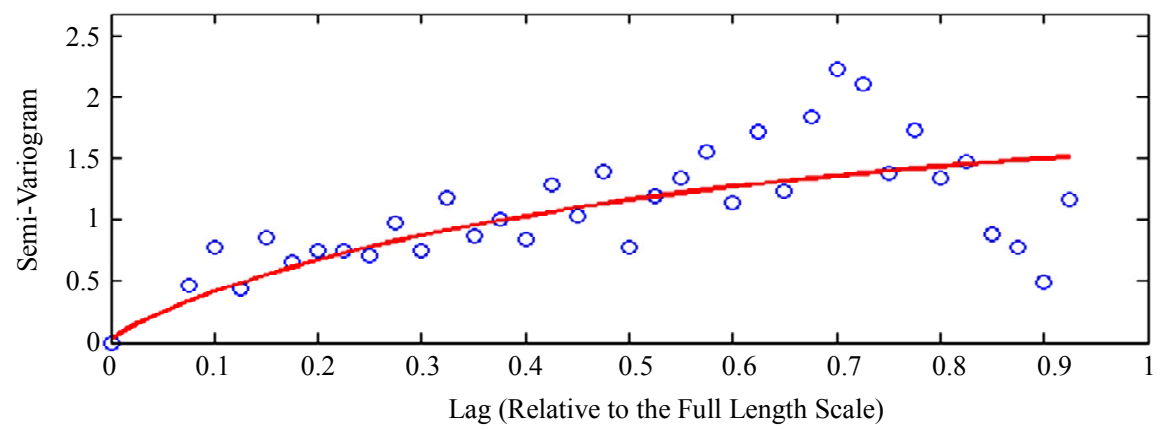

Figure 13. Semivariogram adjustment from theoretical to experimental (120 samples).

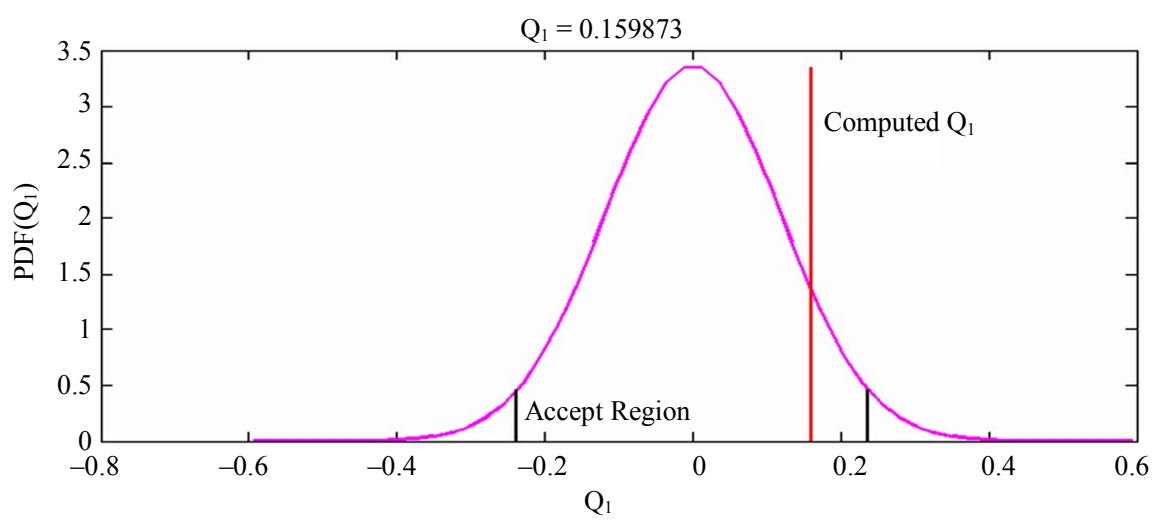

Figure 14. Validation process for 120 samples. 
The area of validity of the interpolation, as expected, is considerably reduced compared to the total area of study as shown in Figure 15, due to the smaller number of samples obtained.

For the third case we have 87 measurements which minimum power level is $-97.634 \mathrm{dBm}$ while the maximum is $-74.697 \mathrm{dBm}$. The area in which samples are distributed is far less than the total study areas shown in Figure 16. What is reflected in the experimental variogram in Figure 17.

However the approximation error Q is minimal; nearby to zero as seen in Figure 18. This is because the power range is much smaller than in the previous cases, therefore the difference between the powers measured in ad- jacent points is less reducing the range of possible values of power at each interpolation.

When the power range is as small as this case is possible to appreciate the change in the level with more clarity, but consequently the area of validity of the estimate is much lower as reflected in the scales of the axes in Figure 19.

Finally, when the data are barely sufficient to generate a theoretical variogram and the separation between them is great, the approximation error will be larger, or sometimes not be in the range of acceptance. When measurements are made at a great distance from the Node B there can be problems like this, accordingly analysis of the radiation pattern is more complicated.

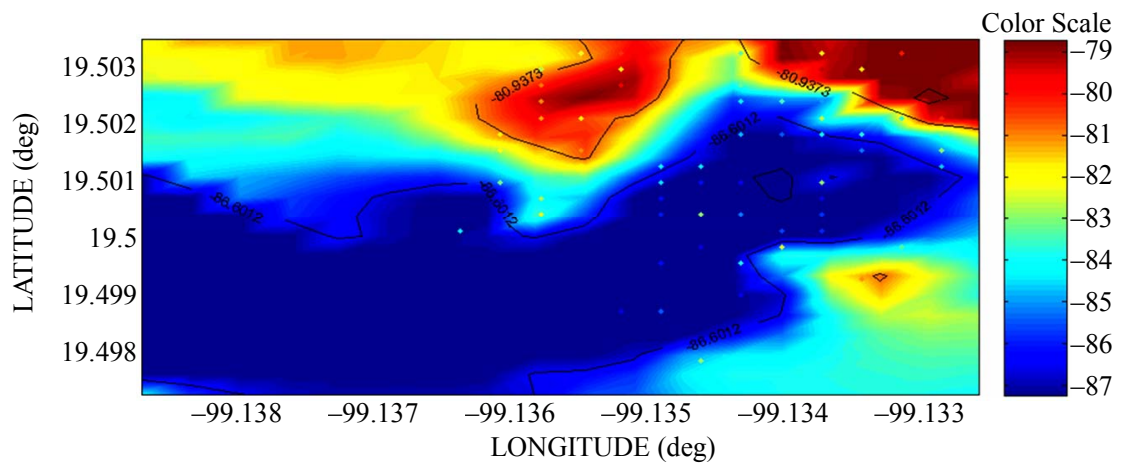

Figure 15. Coverage map for 120 samples.

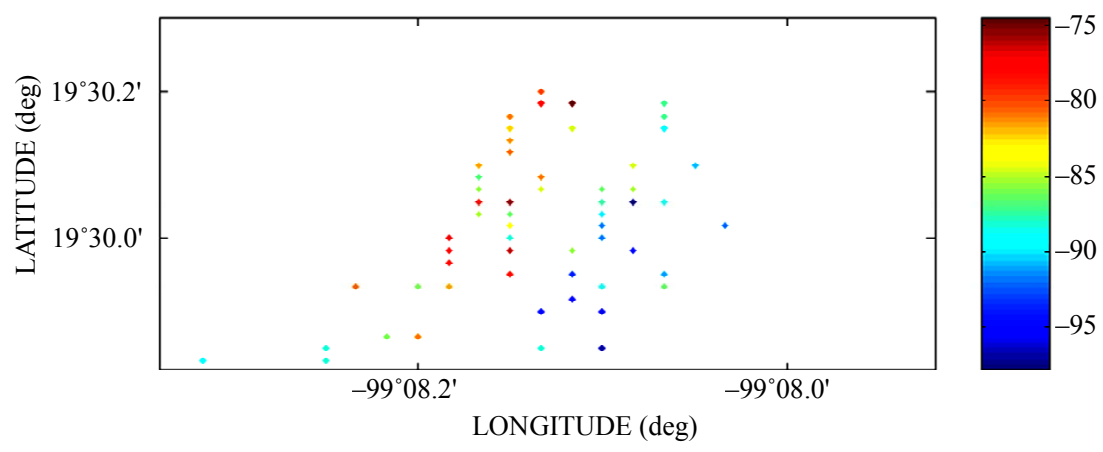

Figure 16. Location of 87 measurements.

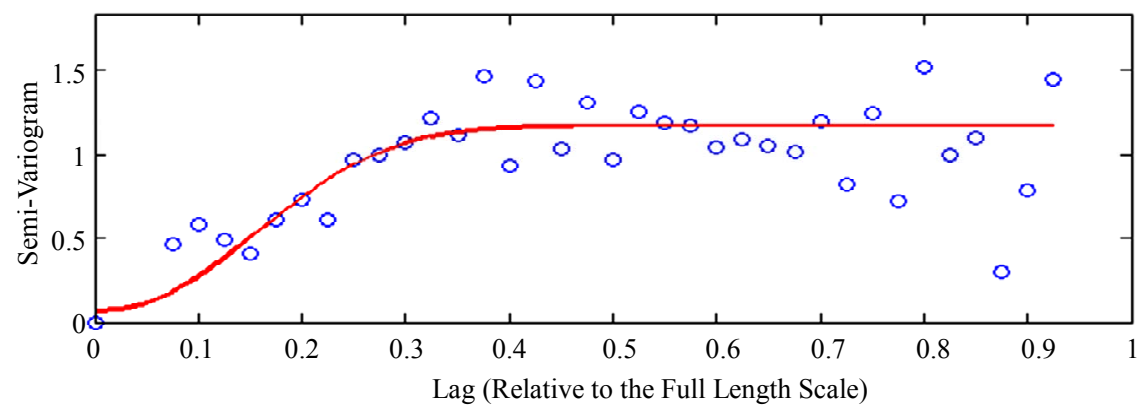

Figure 17. Semivariogram adjustment from theoretical to experimental (87 samples). 


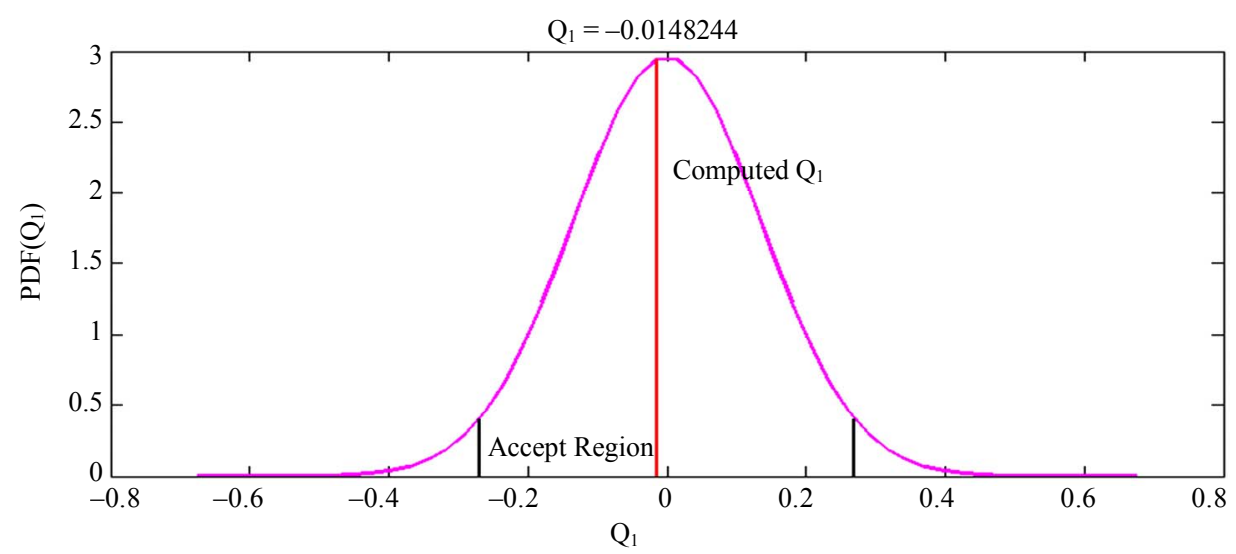

Figure 18. Validation process for 87 samples.

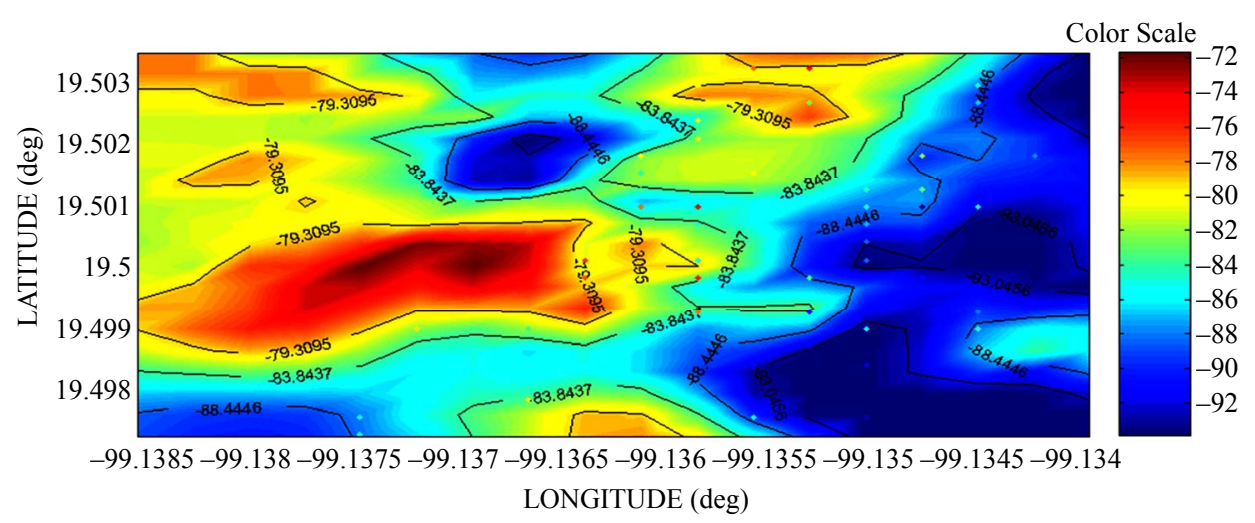

Figure 19. Coverage map for 87 samples.

\section{Conclusions}

Based in the results, is possible concluded that the Krige Method is optimal for power levels analysis in WCDMA, because from point data can be generated continuous coverage maps through a process of interpolation which predicts the power values in places where there is not information based on adjacent values. To create reliable coverage maps is necessary to take into account the following considerations.

- Make sure you have a sufficient number of measurements, which will depend on the total area to study and variable power levels.

- If the power levels are divers, nearby samples allow greater similarity exists between them and ensures that the interpolation will be more reliable in an area very similar to the total.

- Although it is possible to validate a coverage map with a smaller number of samples, this will depend on the operating conditions of the Node B.

- If the level is very variable in the measurement area measurement should be taken as nearby as possible, make sure the position information change, otherwise only have many power values at the same point and it will be unnecessary information for the estimation process.

- If the power levels are not drastic changes can be estimated efficiently without taking a large number of samples.

- To optimize the computing time, data must be arranged in descending or ascending primarily based on the longitude information, later depending on latitude and finally based on power levels. Taking the same order for all the columns in which information is organized. Since the variogram is obtained based on comparisons between pairs of neighboring points and the smaller the difference between them narrower the range of possible power values interpolated between them, obtaining a more efficient estimation.

- Measurements were made at a height between 1.1 and 1.4 meters since it is the average height in which users carry mobile devices.

\section{Acknowledgements}

Authors thank to the IPN for the support received. 


\section{References}

[1] K. Tachikawa, "WCDMA Mobile Communications System," John Wiley \& Sons, Hoboken, 2002.

[2] L. Jaana, W. Achim and N. Tomás, "Radio Network Planning and Optimization for UMTS," 2nd Edition, John Wiley and Sons, Hoboken, 2006.

[3] I. Gual, M. Victoria and S. V. Amelia, "Space-Temporal Models Statistics in Perimetry," Doctoral Thesis, De- partment of Mathematics, School of Technology and Experimental Sciences, Jaume 1 University, Castellón de la Plana, 2003.

[4] J. I. Z. Castro, "Analysis of Pilot Pollution in WCDMA Networks," Masters Thesis, National Polytechnic Institute, México, 2011.

[5] Practical Tips on WCDMA Measurements, Application Note No. 11410-00378, Revision B, United States, 2008. 